\title{
A Nanodrug Consisting Of Doxorubicin And Exosome Derived From Mesenchymal Stem Cells For Osteosarcoma Treatment In Vitro
}

This article was published in the following Dove Press journal:

International Journal of Nanomedicine

\author{
Hongxiang Wei ${ }^{1, *}$ \\ Jinyuan $\mathrm{Chen}^{2, *}$ \\ Shenglin Wang ${ }^{1} *$ \\ Feihuan $\mathrm{Fu}^{3}$ \\ Xia Zhu' \\ Chaoyang $\mathrm{Wu}^{\prime}$ \\ Zhoujie Liu (D) ${ }^{4}$ \\ Guangxian Zhong' \\ Jianhua Lin'
}

'Department of Orthopaedics, The First Affiliated Hospital of Fujian Medical University, Fuzhou 350004, People's Republic of China; ${ }^{2}$ Department of Centralab, The First Affiliated Hospital of Fujian Medical University, Fuzhou 350004, People's Republic of China; ${ }^{3}$ Department of Endocrinology, The County Hospital of Anxi, Anxi 362400, People's Republic of China; ${ }^{4}$ Department of Pharmacy, The First Affiliated Hospital of Fujian Medical University, Fuzhou 350004, People's Republic of China

*These authors contributed equally to this work

Correspondence: Guangxian Zhong; Jianhua Lin

Department of Orthopaedics, The First Affiliated Hospital of Fujian Medical University, Fuzhou 350004, People's Republic of China

Tel/fax +86 59l 87981029

Email zhongguangxian@163.com; jianhual@I26.com
Purpose: The primary goal of the present study was to develop the nano-drug consisting of doxorubicin and exosome derived from mesenchymal stem cells, and to explore its effect on osteosarcoma in vitro.

Methods: The exosomes were isolated from bone marrow MSCs (BM-MSCs) by an Exosome Isolation Kit. The exosome-loaded doxorubicin (Exo-Dox) was prepared by mixing exosome with Dox-HCl, desalinizing with triethylamine and then dialyzing against PBS overnight. The nanoparticle tracking analysis (NTA) and transmission electron microscope (TEM) were used to characterize of the exosome and Exo-Dox. The cytotoxicity of Exo-Dox was determined by CCK-8 assay. Further, the cellular uptake of different drugs was analyzed using inverted fluorescence microscope and flow cytometry.

Results: The typical exosome structures can be observed by TEM. After loading with doxorubicin, its size is larger than free exosome. Compared with the free Dox, the prepared Exo-Dox showed enhanced cellular uptake efficiency and anti-tumor effect in osteosarcoma MG63 cell line but low cytotoxicity in myocardial H9C2 cell line.

Conclusion: The prepared Exo-Dox could be used as an excellent chemotherapeutic drug for treatment of osteosarcoma in vitro. Considering the tumor-homing feature of BM-MSCs, the Exo-Dox may be a good candidate for targeted osteosarcoma treatment in future study.

Keywords: exosome, mesenchymal stem cells, doxorubicin, osteosarcoma

\section{Introduction}

The rapid advance of nanocarriers for drug delivery has led to the emergence of many targeted drugs for cancer treatment. ${ }^{1,2}$ These nanocarriers have some advantages such as specific tumor targeting and accumulation, stabilized drug and easy release in tumor microenvironment. ${ }^{3,4}$ Many materials have been used for preparing nanocarriers, such as nanogold particles, ${ }^{5}$ carbon nanotubes, ${ }^{6}$ micelles and liposomes, ${ }^{7,8}$ polymeric nanoparticles ${ }^{9}$ and polymer conjugates. ${ }^{10}$ Among these materials, lipid-based nanocarriers have attracted increasing attentions due to their good biocompatibility and biodegradability, the ease of breaching the plasma membrane, easy isolation of drugs from the encircling environment, and the ability of entrapping both hydrophilic and hydrophobic capsule. ${ }^{11-13}$ For example, Feng et al. ${ }^{14}$ fabricated cisplatin-prodrugconstructed liposomes with the advantages of precisely defined/easily tunable drug compositions, stealth-like pharmacokinetics, efficient tumor intake, and loading with various types of imaging or therapeutic agents simultaneously. Fang et al. ${ }^{15}$ developed a PEGylated liposomal system loading with SN38 prodrug, which effectively improved 
its systemic circulation and preferential accumulation in tumors. Despite the great successes in efficacy and targeting of these lipid-based nanocarriers, there is still attention needed to be paid in immune rejection by host immune system. ${ }^{16}$ Thus, the natural particulates which have a natural tropism toward target cells with high selectivity and efficiency have been exploited as drug delivery vehicles. These natural particulates include bacteria, viruses, red blood cells, macrophages, lymphocytes, stem cells and exosome, among which exosome is a more promising natural particulate for its wide distribution in biological fluids and intrinsic homing capability. ${ }^{17-19}$

Exosome, as a membrane vesicle with a diameter of 30-100 nm, has a bi-lipid membrane with the same orientation as plasma membrane and contains proteins and genetic materials which play an important role in intercellular communication. ${ }^{20}$ Due to its many excellent attributes including wide distribution in biological fluids, intrinsic homing capability and penetrable to blood-brain barrier, exosome undoubtedly could be an ideal drug delivery vehicle. ${ }^{21-23}$ Many types of cells such as epithelial cells, ${ }^{24}$ hematopoietic cells, ${ }^{25}$ tumor cells ${ }^{26}$ and mesenchymal stem cells (MSCs) ${ }^{27}$ are known to secrete exosome. Among these cells, MSCs are recognized as an ideal cell candidate for the mass production of exosomes because of its good performances such as the ease of isolation from ethically noncontroversial human materials, the large ex vivo expansion capacity, the tropism toward tumor tissue, low immunogenicity and side effect. ${ }^{28-31}$

Motivated by the excellent performances of MSCs and exosome, in this work, the exosomes from bone marrow MSCs (BM-MSCs) were used as nanocarrier to load chemotherapeutic drug doxorubicin (Dox) for osteosarcoma treatment. Compared with the free Dox, the exosome-loading Dox exhibited better cellular uptake and antitumor effects for osteosarcoma. Also, these exosomes from BMMSCs had been demonstrated no cytotoxicity for osteosarcoma cell line, which may make it a good tumor-targeted drug nanocarrier in further study.

\section{Materials And Methods}

\section{Cell Lines And Materials}

The human osteosarcoma cell line MG63 and myocardial H9C2 cell line were purchased from the American Type Culture Collection (ATCC). The mouse bone marrow MSC with stable transfection of GFP was purchased from Cyagen Biosciences Inc (Guangzhou, China), the quality inspection report was done by the company. Phosphate-buffered saline
(PBS) was purchased from HyClone Laboratories (Logan, Utah, USA). Trypsin-EDTA (0.25\%), penicillin-streptomycin, Dulbecco's Modified Eagle Medium (DMEM) and Minimum Essential Medium (MEM) were all obtained from Gibco Life Technologies (Grand Island, NY). Mesenchymal stem cell medium-serum free (MSCM-sf) was from ScienCell (San Diego, California, USA). Triethylamine was purchased from Aladdin Industrial Corporation (Shanghai, China). Doxorubicin (Dox) hydrochloride was purchased from Ourchem (Shanghai, China). D36mm Dialysis belt (800014,000 wt) was from Beijing DingGuo Changsheng Biotechnology (cat. AT-C-026, Beijing, China). Exosome Isolation Kit was purchased from Umibio (UR52121, Shanghai, China). Bicinchonininc acid (BCA) protein assay kit was purchased from Beyotime Biotechnology (Jiangsu, China). Cell lysis buffer (10X), phenylmethanesulfonyl fluoride (PMSF) and Protease Inhibitor Cocktail (100X) were all purchased from Cell Signaling Technology (Danvers, MA, USA). Cell counting kit-8 (CCK-8) was obtained from Dojindo Molecular Technologies (Kumamoto, Japan). Cell Culture Plates were from Fisher Scientific (cat. 310109008-96 Wells, Flat Bottom).

\section{Cell Culture}

Osteosarcoma MG63 cell line and myocardial H9C2 cell line were propagated in a DMEM medium supplemented with $10 \%$ fetal bovine serum (FBS, Hyclone Thermo Scientific, USA) and $1 \%$ penicillin-streptomycin. MSCs were cultured in MEM medium supplemented with $10 \%$ fetal bovine serum (FBS, ScienCell, San Diego, California, USA) and 1\% antibiotics. All cells were maintained at an atmosphere containing $5 \% \mathrm{CO}_{2}$ and maintained at $37^{\circ} \mathrm{C}$ in a humidified chamber (Thermo Fischer Scientific, Waltham, MA, USA), and the medium was routinely replaced every $2-3$ days.

\section{Isolation Of Exosomes}

After growing to $70-80 \%$ confluency, MSCs were cultured in MSCM-sf medium supplemented with 1\% MSCGS-sf (ScienCell, cat. 7562) and 1\% P/S Solution (ScienCell, cat. 0503) for $48 \mathrm{hrs}$. Then, the conditioned medium was collected and exosomes were isolated with Exosome Isolation Kit according to the manufacturer's instructions. Briefly, the conditioned medium was centrifuged at $3000 \mathrm{~g}$ for $10 \mathrm{~min}$ to dislodge cells and debris. The Kit reagents and the conditioned medium were mixed in proportion to 1:4. Then, the mixture was vortexed for $1 \mathrm{~min}$ and incubated for $2 \mathrm{hrs}$ at $4^{\circ} \mathrm{C}$ before centrifugation at $10,000 \mathrm{~g}$ for $60 \mathrm{~min}$ to separate exosomes. After that, $1 \times$ PBS was used 


\section{(A)}

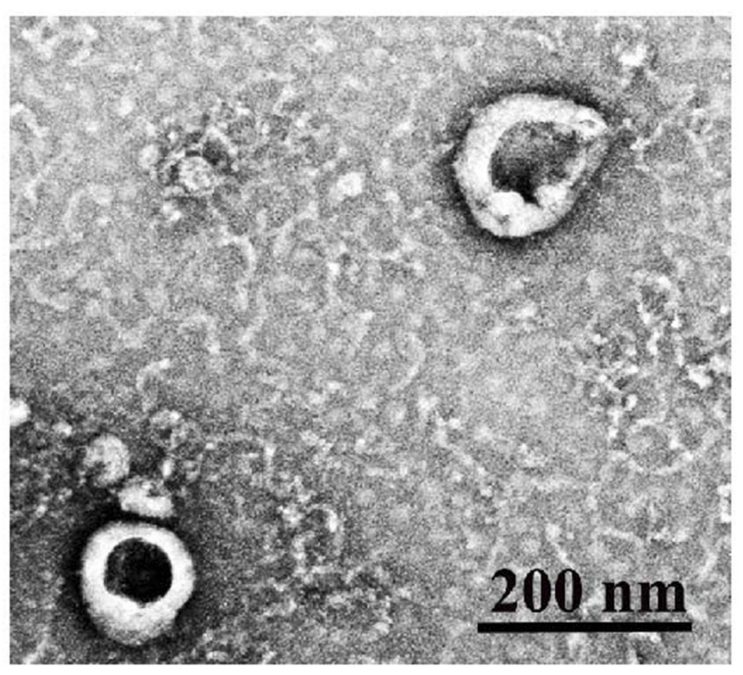

( C)

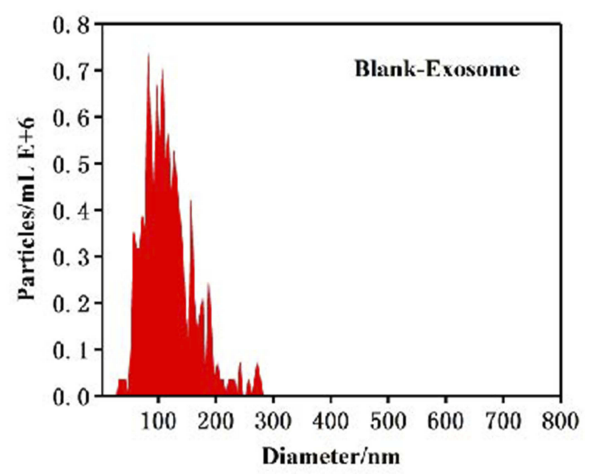

(B)

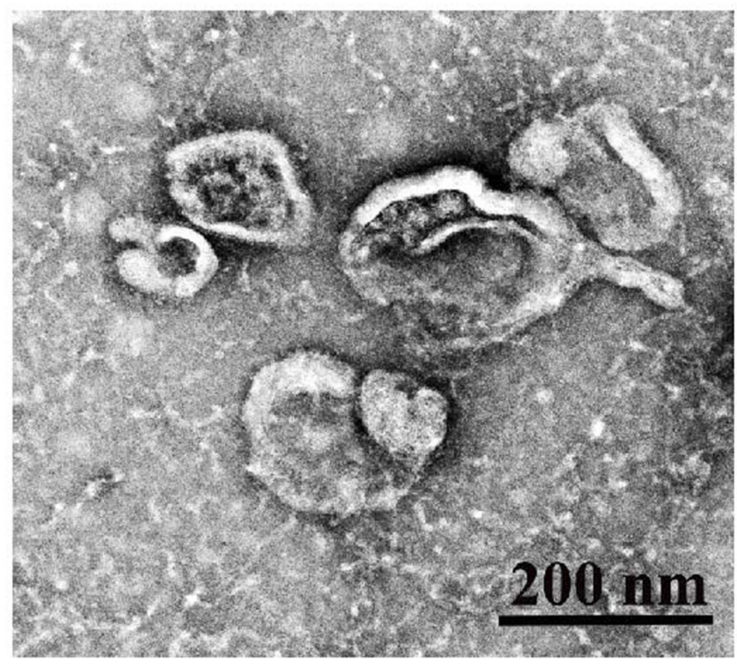

(D)

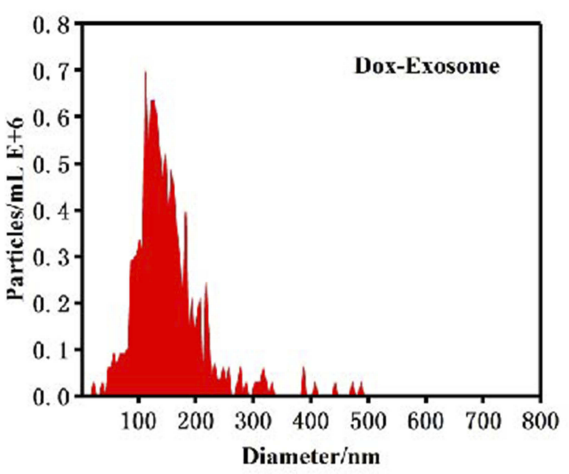

$(\mathrm{E})$

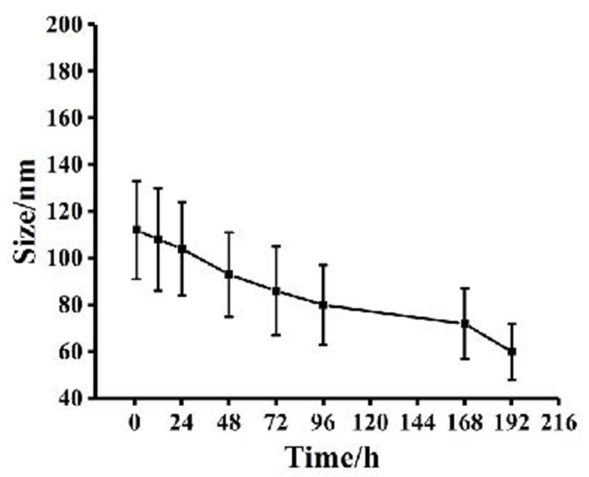

Figure I Characterization of exosomes: the representative TEM image of blank exosome (A) and exosome-doxorubicin (B). Size distributions of blank exosome (C) and exosome-doxorubicin (D) based on NTA measurements. The peak diameters were at II $2.4 \mathrm{~nm}$ for free exosome and I52.7 nm for exosome-doxorubicin. (E) Particle size by nanoparticle tracking analysis for MSCs-derived exosomes during storage at $-20{ }^{\circ} \mathrm{C}$. 
to resuspend exosome-containing pellet, and then centrifuged at $3000 \mathrm{~g}$ for $10 \mathrm{~min}$ in Exosome Purification Filter. All the centrifugation steps were carried out at $4{ }^{\circ} \mathrm{C}$ to maintain the activity of exosomes. The weight of exosome was measured by BCA kit according to the manufacturer's instructions. The isolated exosomes were immediately stored at $-80^{\circ} \mathrm{C}$ until further experiments.

\section{Preparation Of Dox-Loaded Exosome (Exo-Dox) And Drug Release}

Doxorubicin (Dox) hydrochloride was appropriately diluted by sterile water for injection (SWFI). The $70 \mu \mathrm{L}$ of Dox $\mathrm{HCl}\left(1 \mathrm{mg} \mathrm{mL}^{-1}\right)$ was mixed with $930 \mu \mathrm{L}$ exosome solution $\left(1 \mathrm{mg} \mathrm{mL} \mathrm{m}^{-1}\right)$ for $30 \mathrm{~min}$ and desalinized with triethylamine for $1 \mathrm{hr}$ at room temperature (RT). The mix solution was then added to dialysis tube prepared by dialysis belt and centrifuge tube and dialyzed against PBS overnight at $4^{\circ} \mathrm{C}$ to prepare the Exo-Dox complex. The amount of DOX loaded into exosomes was calculated from a standard curve obtained from BioTek ELX800 (Gene Company Limited, USA) by detecting the absorbance value at the wavelength of $490 \mathrm{~nm}$.

In order to measure the in-vitro drug release profile, 3 $\mathrm{mL}$ of Exo-Dox solution was transferred into dialysis tubes immersed in PBS at $\mathrm{pH} 7.4$ or $\mathrm{pH}$ 4.5. The release of Dox was performed at $37^{\circ} \mathrm{C}$ and different time intervals. At 1,2 , $4,8,12,24$ and $36 \mathrm{~h}$, the concentrations of Dox were determined based on calibration curve by Bioequivalence ELx800 automated enzyme immunoassay analyzer.

\section{Characterization Of The Exosome And Exo-Dox}

After purification of exosomes and preparation of ExoDox, the hydrodynamic size and concentration of samples were measured using nanoparticle tracking analysis (NTA) with Zeta View PMX 110 (Particle Metrix, Meerbusch, Germany) and corresponding software Zeta View 8.04.02.

The sample solutions were fixed on Formvar-carbon copper grids and were negatively stained for $2 \mathrm{~min}$ and airdried for morphologic visualization using a transmission electron microscope (TEM, HT7700, Hitachi, Japan).

\section{Cytotoxicity Of Exo-Dox In Vitro}

The cytotoxicity of Exo-Dox was determined by CCK-8 assay. MG-63 cells and $\mathrm{H} 9 \mathrm{C} 2$ cells were seeded into 96well plates at a density of 3000 or 5000 cells/well and were cultured at $37^{\circ} \mathrm{C}$ for $24 \mathrm{hrs}$. Then, the cells were treated with exosomes $\left(0,5,10,20,100\right.$ and $\left.200 \mu \mathrm{g} \mathrm{mL}^{-1}\right)$ and Dox formulations (Dox and Exo-Dox) with the equivalent Dox concentrations of $0,0.25,0.5,1,5,10$, $20,50,100 \mu \mathrm{g} \mathrm{mL}{ }^{-1}$ for $24 \mathrm{hrs}$. After that, the cells were incubated with CCK-8 solution for $2 \mathrm{hrs}$, then the optical density (OD) at $450 \mathrm{~nm}$ was detected.

\section{Cell Uptake And Localization}

In order to detect the cellular uptake of different drugs, MG63 cell and $\mathrm{H} 9 \mathrm{C} 2$ cell were incubated with Dox and Exo-Dox for $1 \mathrm{hr}, 4 \mathrm{hrs}$ and $24 \mathrm{hrs}$. Cells without any treatment were used as control. After incubation, cells were washed three times with PBS and fixed in 4\% paraformaldehyde for 15 min, the cell nuclei were labelled by using DAPI or antifluorescence quenching seal solution (containing DAPI). Finally, the localization of Exo-Dox and Dox in cells was visualized by inverted fluorescence microscope (Carl Zeiss Microscope Systems, Jena, Germany). To further investigate the cellular uptake process of Dox and Exo-Dox. MG63 cell and $\mathrm{H} 9 \mathrm{C} 2$ cell were seeded into 12 -well plates at a density of $1 \times 10^{5}$ cells/well and were cultured for $24 \mathrm{~h}$. The cells were also incubated with Dox and Exo-Dox for 1, 4 and 24 hrs. Then, the cells were washed twice with PBS, and cell pellets were collected by centrifugation at 1000rpm for $5 \mathrm{~min}$. The fluorescence signals in cells were analyzed using flow cytometry (BD Accuri C6, USA).

\section{Statistical Analysis}

Statistical analysis of data was performed using Student's $t$-test and one-way analysis of variance (ANOVA). Probability values lower than 0.05 were considered statistically significant.

\section{Results And Discussion Characterization Of Exosome Derived From BM-MSCs And Dox-Loaded Exosome}

The morphological investigation of exosomes before and after loading with Dox was performed by transmission electron microscope (TEM). As shown in Figure 1A and B, the typical exosome structures can be observed. Nanoparticle tracking analysis (NTA) (Figure 1C and D) indicated that the exosomes had a relatively narrow size distribution, and the peak diameters were at $112.4 \mathrm{~nm}$ for free exosome. After loading with Dox, the peak diameters increased to $152.7 \mathrm{~nm}$. The stability of these exosomes over time was also investigated by NTA (Figure 1E), and the results showed that the size did not change significantly within 7 days at $-20^{\circ} \mathrm{C}$. 


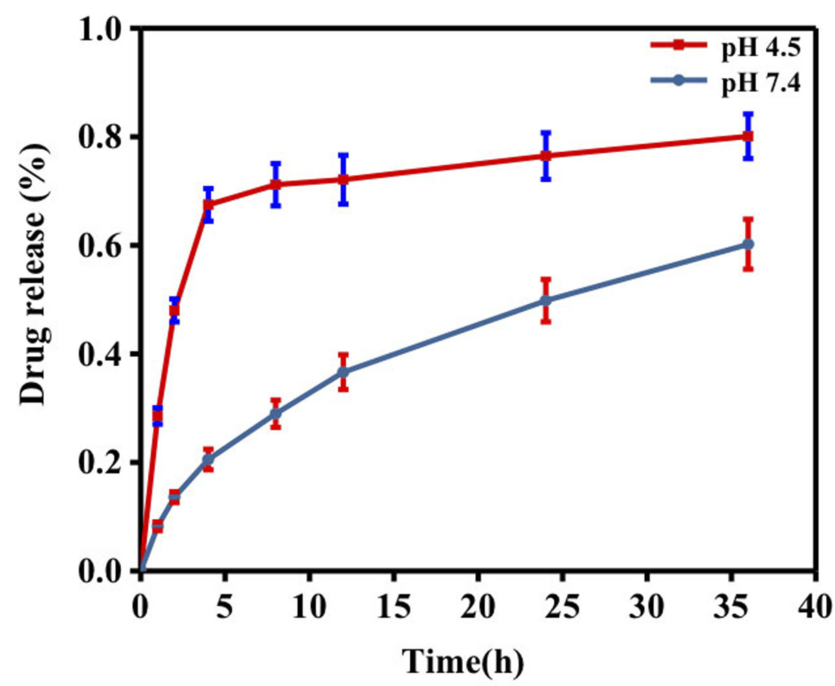

Figure 2 Drug release profile of Exosome-Doxorubicin in PBS with $\mathrm{pH} 4.5$ and $\mathrm{pH}$ 7.4, respectively.

\section{Drug Encapsulation Efficiency And Release Profile}

The drug encapsulation efficiency is an important parameter to evaluate the drug delivery potential of a drug delivery carrier, so it is needed to test the drug encapsulation efficiency of Exo-drug. After calculation, the drug encapsulation efficiency was about $12 \%$ when the mass ratio of Exo/Dox was 93:7.

To investigate in-vitro drug release profile of Exo-Dox, we determined the release of Dox when putting the ExoDox into PBS dialysate with $\mathrm{pH}$ of 7.4 or 4.5 (Figure 2) which mimicked the physiological environment and endolysosomal compartment interior environment. ${ }^{32}$ In PBS dialysate with $\mathrm{pH} 7.4$, the release of Exo-Dox was about $60 \%$ after 36 hrs, while in PBS dialysate with $\mathrm{pH} 4.5$, the release rate of Exo-Dox boosted rapidly and achieved about $80 \%$, suggesting that the decrease of the $\mathrm{pH}$ could result in the protonation of DOX and accelerate the release of Dox. The drug release profile of Exo-Dox indicated that the acidic environment, such as late endosomes and lysosomes of cancer cells, can accelerate the Dox release of Exo-Dox, this characteristic may make it a good targeted drug in cancer treatment in further study.

\section{The Cellular Uptake Of Exo-Dox In Vitro} The cellular uptake profile of Exo-Dox was evaluated. Because the MSCs were stably transfected by GFP, the exosomes from the MSCs also had the GFP. After incubating MG 63 cells with free Dox and Exo-Dox for $1 \mathrm{hr}$, the fluorescence microscope observation indicated that all the
(A)

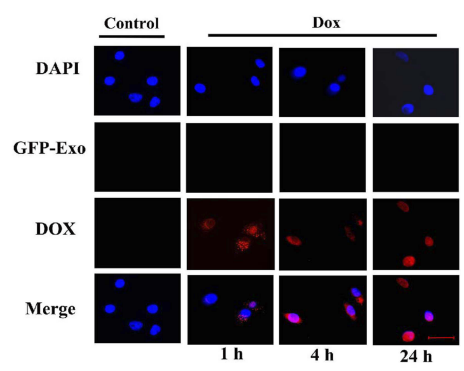

(B)

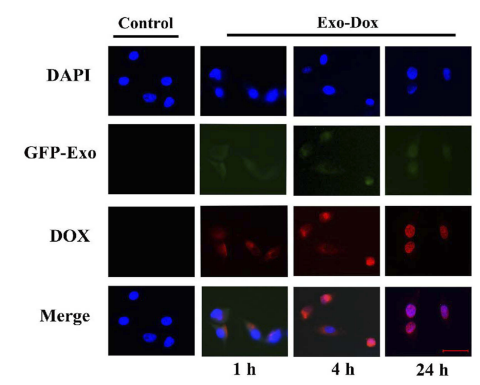

(C)
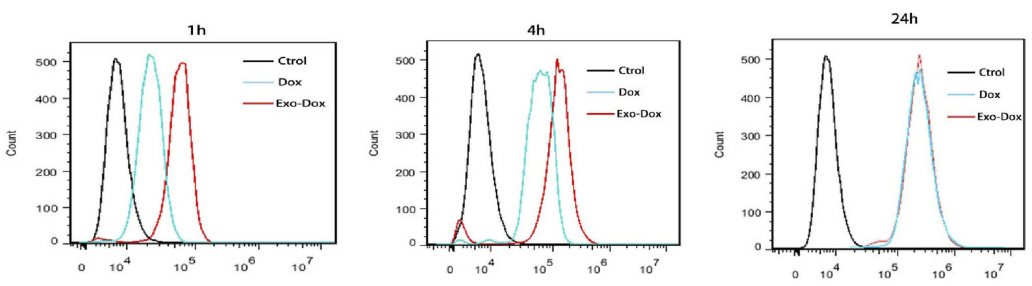

(D)

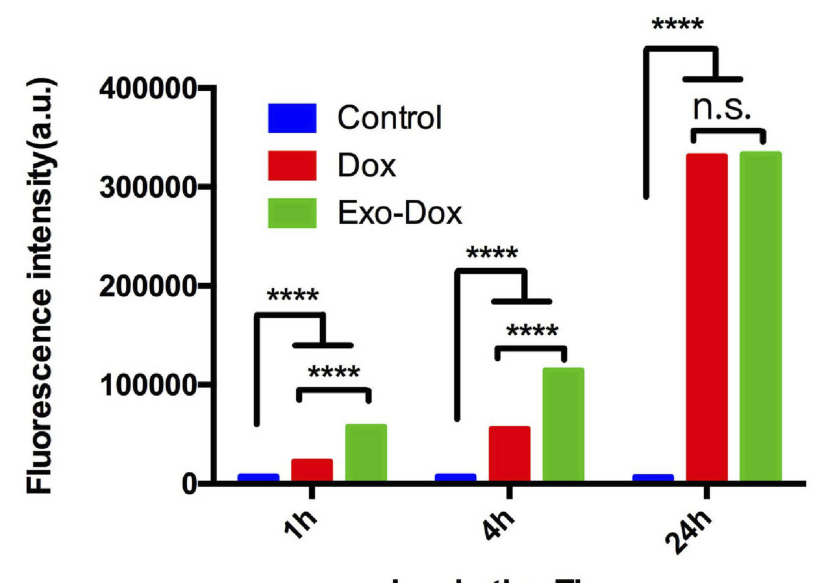

Incubation Time

Figure 3 Intracellular distribution of Dox (A) and Exo-DOX (B) after I hrs, 4 hrs and 24 hrs incubation with MG63 cells, bar was $50 \mu m$. The histogram (C) and Quantification (D) of internalized Dox, Exo-Dox using flow cytometry after incubation with MG63cells for I h,4 h and $24 \mathrm{~h}$. All of the data are presented as mean \pm s.d. ( $\mathrm{n}=3$ ) and analyzed with two-way ANOVA $(* * * * \mathrm{P}<0.000 \mathrm{I})$. 
Dox and Exo-Dox entered into cell (Figure 3A and B), and the exosome (green) and Dox (red) were mostly co-localized (Figure 3B), suggesting that the exosome and Dox could simultaneously enter into the MG63 cells and the exosome can retain the intact structure after entering into cells. After incubation with MG 63 cells for 4 hrs, it was observed more Exo-Dox entered into nucleus comparing with free Dox (Figure 3B), showing that the cellular uptake efficiency of Exo-Dox was higher than that of free Dox at the incubation time of $4 \mathrm{hrs}$, which could be attributed to the easy binding of MSCs-derived exosome with MG63 cell. After incubation with MG 63 cells for 24 hrs, almost all the free Dox and Exo-Dox entered into the nucleus.

(A)

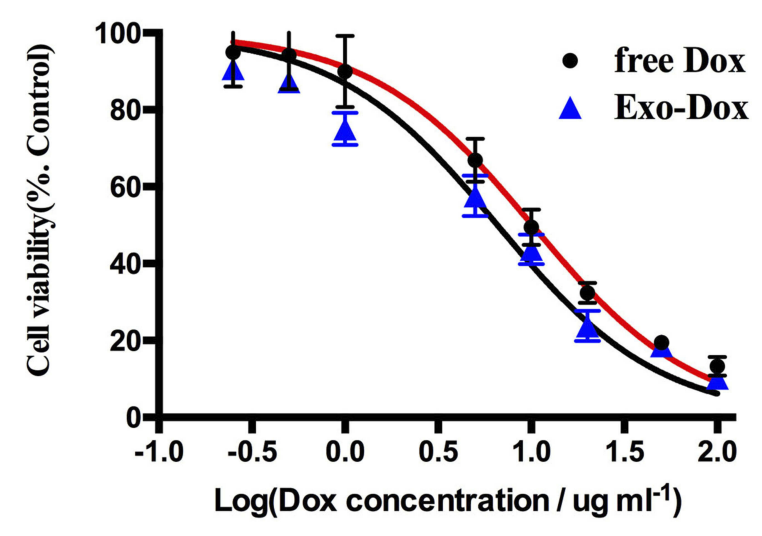

The FACS of cellular uptake of Exo-Dox and free Dox by MG63 cell were also investigated as shown in Figure $3 \mathrm{C}$ and D. From these two figures, we can see that fluorescence intensity of Exo-Dox is much stronger than that of free Dox, showing that more Exo-Dox entered into cell comparing with free Dox. These results were consistent with the results of fluorescence microscope observation.

Because the major side effect induced by doxorubicin is cardiac toxicity, the uptake of Exo-Dox and free Dox by myocardial H9C2 cell was also investigated using inverted fluorescence microscope and flow cytometry. The results showed that fluorescence of Exo-Dox is much weaker than that of free Dox, indicating that less Exo-Dox entering into

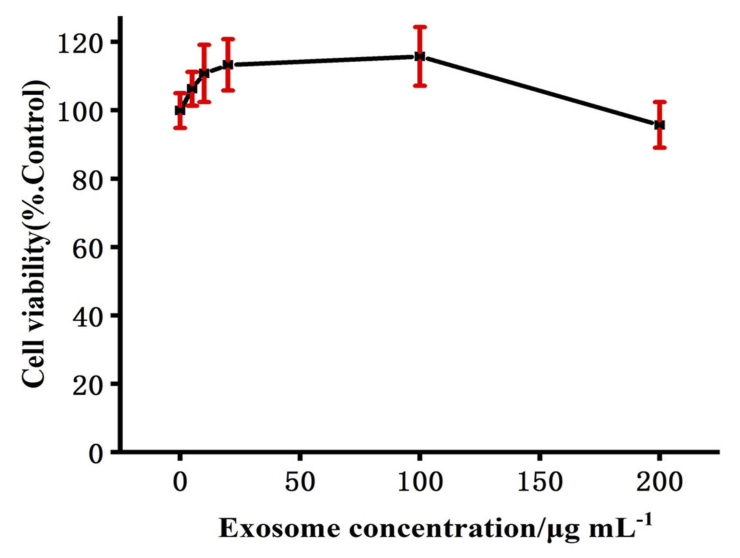

(C)

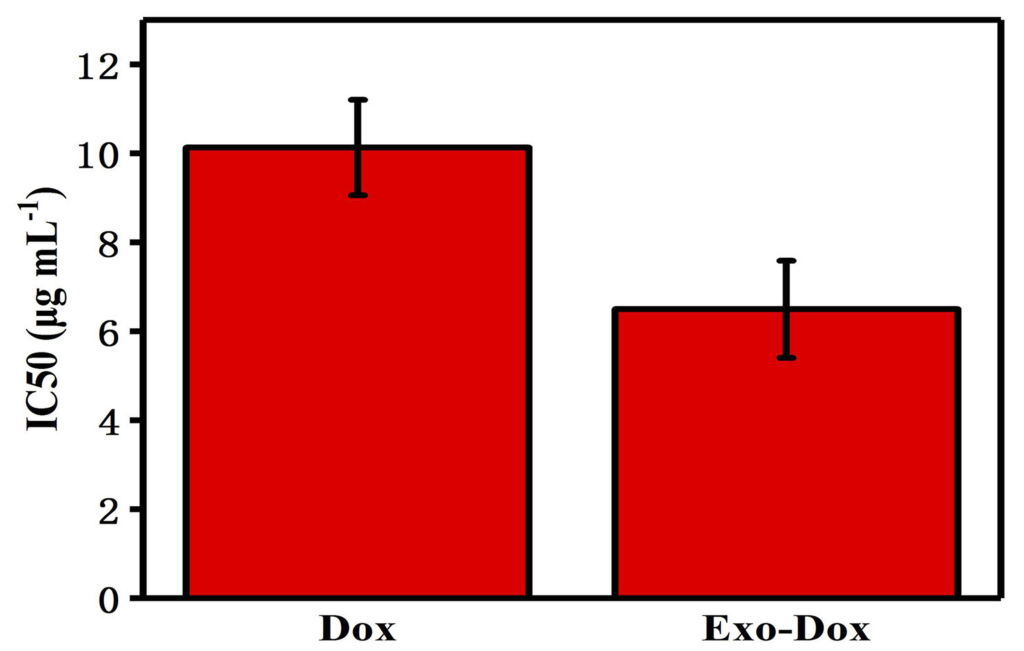

Figure 4 Cell viability of MG63 cells exposed to different concentrations of free doxorubicin, exosome-doxorubicin (A) and blank exosome (B). The IC50 of free doxorubicin and exosome-doxorubicin (C), where the value was calculated according to Figure $\mathrm{A}$. 
cell comparing with free Dox (Figure S1). The opposite results indicated that the uptake of Exo-Dox is easier by MG63 than H9C2 cell.

\section{The Cytotoxicity And Antitumor Effects Of Exo-Dox In Vitro}

The cytotoxic effects of Exo-Dox on osteosarcoma MG63 cell and myocardial H9C2 cell were examined by Cell Counting Kit-8 (CCK-8) cell viability assay (Figure 4). As shown in Figure 4B, the cell viability was maintained as high as $92 \%$ after incubating with free exosome at the concentration of $200 \mu \mathrm{g} \mathrm{mL}^{-1}$ for $24 \mathrm{hrs}$, demonstrating that the exosome had an excellent cytocompatibility.

To further investigate the antitumor effect of Exo-Dox in vitro, MG63 cells were treated with free Dox and ExoDox with the same concentration of Dox for $24 \mathrm{~h}$ followed by CCK-8 assay (Figure 4A). The half-maximal inhibitory concentrations (IC50) of Dox, Exo-Dox were calculated to be 10.13 and $6.48 \mu \mathrm{g} \mathrm{mL}^{-1}$, respectively (Figure 4C). The tumor cell inhibition was in accordance with the cellular uptake efficiency at $4 \mathrm{hrs}$.

Moreover, we then further investigated the cytotoxicity of Dox and Exo-Dox on myocardial H9C2 cell line. As shown in Figure S2, the IC50 value of Exo-Dox is much higher than that of free Dox $(29 \mu \mathrm{g} \mathrm{mL}-1$ for Exo-Dox and $7.312 \mu \mathrm{g} \mathrm{mL}-1$ for Dox, respectively), indicating that the cytotoxicity of Exo-Dox is much weaker for the myocardial cell comparing with free Dox, which is the main priority to use exosome as nanocarrier.

Together, all the results mentioned above, we can see that the Exo-Dox showed selectively anti-tumor effect while had low cytotoxicity for myocardial cells, this may be attributed to the interaction of the membrane proteins in the surface of MSCs-derived exosomes and MG63 cells.

\section{Conclusion}

In summary, this work reported that the exosome isolated from MSCs could be used as a drug nanocarrier to load chemotherapeutic drug Dox. The exosome showed low cytotoxicity, and the Exo-Dox could be taken up by MG63 cells and induce cell death. Compared with free Dox, the Exo-Dox can kill the osteosarcoma cells more effectively and show low cytotoxicity in myocardial cells. Considering the tropism toward tumor tissue of MSCs, the exosome derived from BM-MSCs may provide a promising delivery vehicle for tumor-targeted treatment in further study.

\section{Acknowledgments}

The authors gratefully acknowledge the financial support of Joint Funds for the Innovation of Science and Technology, Fujian Province (2016Y9019), the Outstanding Youth Fund of Fujian Province (2017J06017), the Special Fund of Youth Top-Notch Innovative Talents of Fujian Province (SQNBJ201601) and Trauma Medical Center of Fujian Province.

\section{Disclosure}

The authors report no conflicts of interest in this work.

\section{References}

1. Ji T, Zhao Y, Ding Y, Nie G. Using functional nanomaterials to target and regulate the tumor microenvironment: diagnostic and therapeutic applications. Adv Mater. 2013;25:3508-3525. doi:10.1002/adma.201 300299

2. Ding Y, Li S, Nie G. Nanotechnological strategies for therapeutic targeting of tumor vasculature. Nanomedicine. 2013;8:1209-1222. doi:10.2217/nnm.13.106

3. Ji T, Li S, Zhang Y, et al. An MMP-2 responsive liposome integrating antifibrosis and chemotherapeutic drugs for enhanced drug perfusion and efficacy in pancreatic cancer. ACS Appl Mater Interfaces. 2016;8:3438-3445. doi:10.1021/acsami.5b11619

4. Li S, Zhang Y, Wang J, et al. Nanoparticle-mediated local depletion of tumour-associated platelets disrupts vascular barriers and augments drug accumulation in tumours. Nat Biomed Eng. 2017;1:667679. doi:10.1038/s41551-017-0115-8

5. Ali I, Mukhtar SD, Lone MN, Ali HS, Aboul-Enein HY. Recent advances in mesoporous silica and gold based nanovectors in anticancer drug delivery system. Curr Org Chem. 2017;21(23):24002415. doi:10.2174/1385272820666161025161853

6. Son KH, Hong JH, Lee JW. Carbon nanotubes as cancer therapeutic carriers and mediators. Int J Nanomedicine. 2016;11:5163-5185. doi:10. 2147/IJN.S112660

7. Cagel M, Tesan FC, Bernabeu E, et al. Polymeric mixed micelles as nanomedicines: achievements and perspectives. Eur J Pharm Biopharm. 2017;113:211-228. doi:10.1016/j.ejpb.2016.12.019

8. Andriyanov AV, Portnoy E, Koren E, et al. Therapeutic efficacy of combined PEGylated liposomal doxorubicin and radiofrequency ablation: comparing single and combined therapy in young and old mice. J Controlled Release. 2017;257:2-9. doi:10.1016/j.jconrel. 2017.02.018

9. Banik BL, Fattahi P, Brown JL. Polymeric nanoparticles: the future of nanomedicine. WIREs Nanomed Nanobiotechnol. 2016;8(2):271299. doi:10.1002/wnan.1364

10. Cai H, Wang XJ, Zhang H, et al. Enzyme-sensitive biodegradable and multifunctional polymeric conjugate as theranostic nanomedicine. Appl Mater. 2018;11:207-218. doi:10.1016/j.apmt.2018.02.003

11. Esfanjani AF, Assadpour E, Jafari SM. Improving the bioavailability of phenolic compounds by loading them within lipid-based nanocarriers. Trends Food Sci Technol. 2018;76:56-66. doi:10.1016/j.tifs. 2018.04.002

12. Kim CH, Lee SG, Kang MJ, Lee S, Choi YW. Surface modification of lipid-based nanocarriers for cancer cell-specific drug targeting. $J$ Pharm Invest. 2017;47(3):203-227. doi:10.1007/s40005-017-0329-5

13. Dolatabadi JEN, Omidi Y. Solid lipid-based nanocarriers as efficient targeted drug and gene delivery systems. TrAC Trends Anal Chem. 2016;77:100-108. doi:10.1016/j.trac.2015.12.016 
14. Feng LZ, Gao M, Tao DL, et al. Cisplatin-prodrug-constructed liposomes as a versatile theranostic nanoplatform for bimodal imaging guided combination cancer therapy. Adv Funct Mater. 2016;26:22072217. doi:10.1002/adfm.201504899

15. Fang T, Dong YH, Zhang XM, Xie K, Lin L, Wang HX. Integrating a novel SN38 prodrug into the PEGylated liposomal system as a robust platform for efficient cancer therapy in solid tumors. Int $J$ Polym Mater Polym Biomater. 2018;67(6):39-48.

16. Yoo JW, Irvine DJ, Discher DE, Mitragotri S. Bio-inspired, bioengineered and biomimetic drug delivery carriers. Nat Rev Drug Discov. 2011;10:521-535. doi:10.1038/nrd3499

17. Simons M, Raposo G. Exosomes- vesicular carriers for intercellular communication. Curr Opin Cell Biol. 2009;21:575-581. doi:10.1016/ j.ceb.2009.03.007

18. van den Boorn JG, Schlee M, Coch C, Hartmann G. SiRNA delivery with exosome nanoparticles. Nat Biotechnol. 2011;29:325-326. doi:1 $0.1038 /$ nbt. 1830

19. Lakhal S, Wood MJA. Exosome nanotechnology: an emerging paradigm shift in drug delivery. Bioessays. 2011;33:737-741. doi:10.1002/ bies. 201100076

20. Théry C, Zitvogel L, Amigorena S. Exosomes: composition, biogenesis and function. Nat Rev Immunol. 2002;2(8):569-579. doi:10.1038/nri855

21. Simpson RJ, Jensen SS, Lim JW. Proteomic profiling of exosomes: current perspectives. Proteomics. 2008;8:4083-4099. doi:10.1002/ pmic. 200800109

22. Hood JL, San RS, Wickline SA. Exosomes released by melanoma cells prepare sentinel lymph nodes for tumor metastasis. Cancer Res. 2011;71:3792-3801. doi:10.1158/0008-5472.CAN-10-4455

23. Alvarez-Erviti L, Seow Y, Yin H, Betts C, Lakhal S, Wood MJA. Delivery of siRNA to the mouse brain by systemic injection of targeted exosomes. Nat Biotechnol. 2011;29:341-345. doi:10.1038/ nbt. 1807
24. Richesa A, Campbell E, Borger E, Powis S. Regulation of exosome release from mammary epithelial and breast cancer cells - a new regulatory pathway. Eur J Cancer. 2014;50(5):1025-1034. doi:10. 1016/j.ejca.2013.12.019

25. Hornick NI, Doron B, Abdelhamed S, et al. AML suppresses hematopoiesis by releasing exosomes that contain microRNAs targeting c-MYB. Sci Signal. 2016;9(44):ra88. doi:10.1126/scisignal.aaf2797

26. Hoshino A, Costa-Silva B, Lyden D. Tumour exosome integrins determine organotropic metastasis. Nature. 2015;527:329-335. doi:10.1038/nature 15756

27. Liang XL, Zhang LN, Wang SH, Han Q, Zhao RC. Exosomes secreted by mesenchymal stem cells promote endothelial cell angiogenesis by transferring miR-125a. J Cell Sci. 2016;129:2182-2189. doi:10.1242/jcs. 170373

28. Lai RC, Arslan F, Tan SS, et al. Derivation and characterization of human fetal MSCs: an alternative cell source for large-scale production of cardioprotective microparticles. J Mol Cell Cardiol. 2010;48:12151224. doi:10.1016/j.yjmcc.2009.12.021

29. Yeo RWY, Lai RC, Zhang B, et al. Mesenchymal stem cell: an efficient mass producer of exosomes for drug delivery. Adv Drug Deliv Rev. 2013;65:336-341. doi:10.1016/j.addr.2012.07.001

30. Kidd S, Spaeth E, Dembinski JL, et al. Direct evidence of mesenchymal stem cell tropism for tumor and wounding microenvironments using in vivo bioluminescent imaging. Stem Cells. 2009;27:26142623. doi:10.1002/stem.v27:10

31. Rezaie Z, Ardeshirylajimi A, Ashkezari MD. Improved anticancer properties of stem cells derived exosomes by prolonged release from PCL nanofibrous structure. Gene. 2018;665:105-110. doi:10.1016/j. gene.2018.05.003

32. Mindell JA. Lysosomal acidification mechanisms. Annu Rev Physiol. 2012;74:69-86. doi:10.1146/annurev-physiol-012110-142317
International Journal of Nanomedicine

\section{Publish your work in this journal}

The International Journal of Nanomedicine is an international, peerreviewed journal focusing on the application of nanotechnology in diagnostics, therapeutics, and drug delivery systems throughout the biomedical field. This journal is indexed on PubMed Central, MedLine, CAS, SciSearch ${ }^{\mathbb{}}$, Current Contents ${ }^{\mathbb{R}} /$ Clinical Medicine,
Journal Citation Reports/Science Edition, EMBase, Scopus and the Elsevier Bibliographic databases. The manuscript management system is completely online and includes a very quick and fair peer-review system, which is all easy to use. Visit http://www.dovepress.com/ testimonials.php to read real quotes from published authors. 